\title{
Popping the genetics bubble
}

$\mathrm{T}$ he hype over the promise of genetics in medicine, like the superlatives that swirl around most "revolutions" in health care, started out as genuine enthusiasm for promising technologies. It didn't take long, however, for researchers to come under pressure to make their work sound exciting, commercializable and immediately applicable. So research institutes and fundraising bodies began touting the promise of a brighter tomorrow in easy-to-digest language.

That fed into the always-hungry media machine, bringing about headlines bloated with sunny adjectives: groundbreaking, breakthrough, gamechanging. Soon the "brave new world" of genetics seeped into sci-fi movies and other areas of pop culture. And let's not forget private companies offering direct-to-consumer genetic testing, some with marketing campaigns best described as, ahem, creative.

All aboard the hyperbole express.

Next stop: health care utopia.

Or not.

Many of the promises made by genomic evangelists have not come to fruition. Still, the field holds much promise, say geneticists, who worry there'll be an inevitable backlash that will slow progress in integrating genomics into medical practice. And though some parties to blame for the hype may be more interested in profit than in improving health, most people swept up in the excitement had good intentions, says Dr. James Evans, editorin-chief of Genetics in Medicine and Bryson Distinguished Professor of Genetics and Medicine at the University of North Carolina in Chapel Hill.

"There is a dramatic alignment of interests that all work together to inflate things without any intent to be nefarious when a bubble like this grows," says Evans. "We need to learn from those bubbles. We need to look at the next big thing, be it nanoparticles or something else, and take a reasonable view of its promise."

Evans and several colleagues have

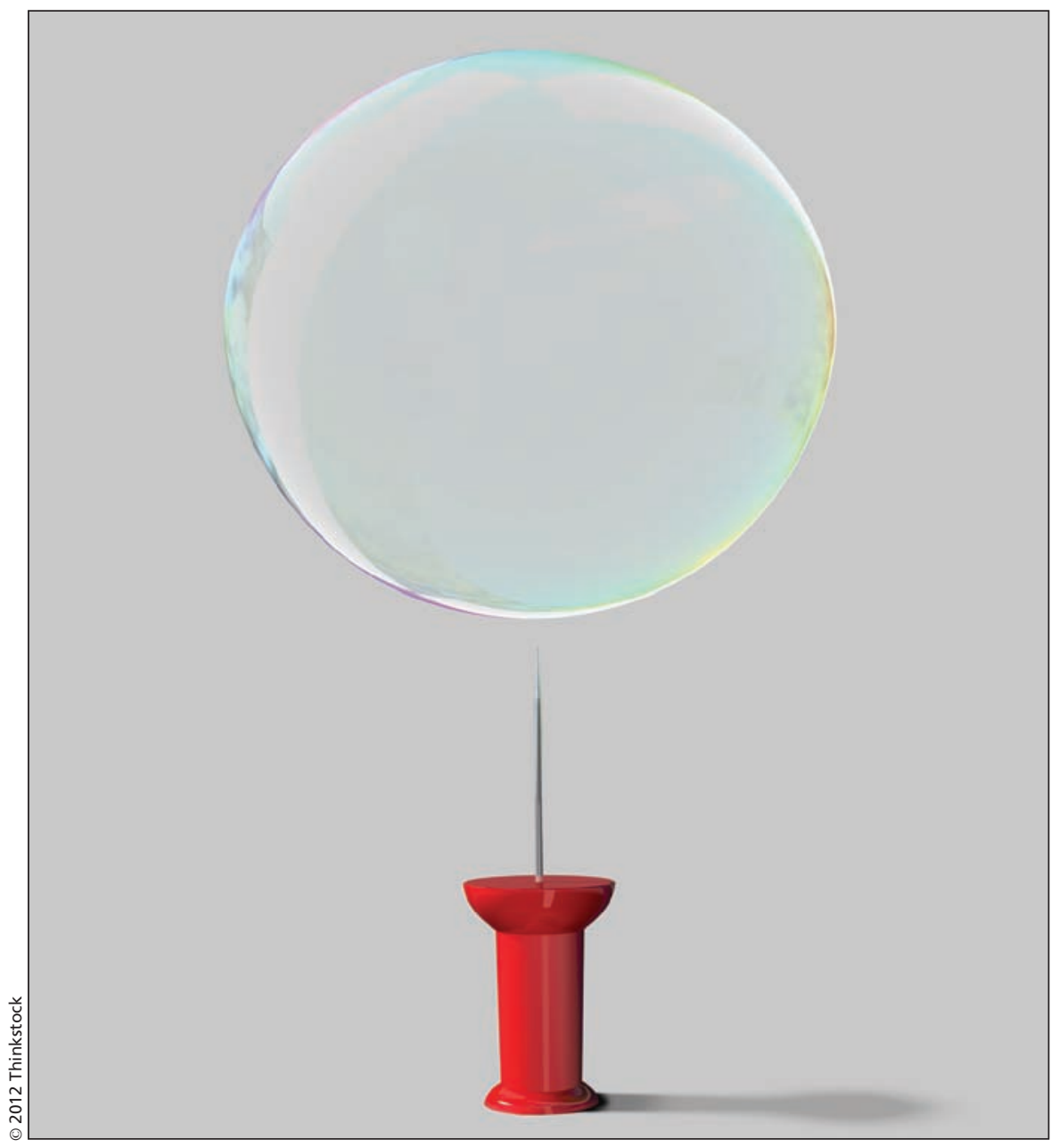

Overhyping the benefits of genomics led to a bit of a backlash once the hype bubble burst.

suggested that exaggerated expectations stemming from a failure to realistically evaluate the potential for genomics to improve human health will "undermine its legitimacy, threaten its sustainability, and result in misallocations of resources" (Science 2011;331:861-2). To get discussions on genetics in medicine back on track, they recommended fostering a realistic understanding of the "incremental nature of science," maintaining focus on acquiring evidence before attempting to change medical practice, and re-evaluating funding priorities to ensure more work is done in areas that may yield practical benefit.

One of the main reasons that genomics did not — and likely will not
- revolutionize medicine is that genetic testing has not proven to be highly predictive of health risks. Genetics is but one component in understanding disease, and most health care problems are caused by many factors, including economic, social and environmental contributors. "All the genetics in the world isn't going to solve our problems," says Evans. "Our job is not to push genetics into medicine but to pull it in when it has been shown to benefit patients."

As for the notion that genetics would usher in an era of personalized medicine, where health care is tailored to each and every individual genome - well, don't get your hopes up, Evans says. "Let's just say I find that overly optimistic. ... 
The idea that it's going to provide a crystal ball that will personalize everyone's care is absurd. It will guide some important medical decisions and will be an important tool in our arsenal."

Of course, promises of personalized medicine don't seem so crazy in comparison with the outlandish claims occasionally made by some direct-to-consumer genetic testing companies. DNA testing has been offered to develop personalized perfumes and tailored diets, and even to assist the lovelorn in finding an exquisite, biologically compatible mate.

"While the desire for profit lies at the heart of this phenomenon, it must be recognized that it builds on the hype about the health value of genetic testing that often flows from the research community, the representations of hype that appears in the media and throughout popular culture, and the public expectations that are fueled by this hype," Timothy Caulfield, a Canada Research Chair in Health Law and Technology who teaches in the law faculty and school of public health at the University of Alberta in Edmonton, wrote in a commentary (JCOM 2011;10:C02).

"In the short term, everybody benefits from the hype," says Caulfield, adding that the abundance of unrealistic expectations shouldn't detract from the actual promise of genomics. "I have no doubt that we are going to see real benefits from the tremendous genetic research that's happening."

Actually, some of that research is already set for prime time, says Cinnamon Bloss, the lead investigator of the Scripps Genomic Health Initiative at the Scripps Translational Science Institute in La Jolla, California. "There are some aspects of genomics that are ready to help improve human health, and one area is pharmacogenics," says Bloss, referring the practice of assessing how individual genotypes will react to med- ication. "This will enable us to better dose a drug so it would better work for a person. If you know a person's genotype, you will also be better able to tell if they might have an adverse event."

Bloss adds that criticism of a genetic service as being clinically irrelevant doesn't mean it lacks value for patients, citing the concept of "personal utility." Even if, for example, a test reveals a predisposition for a condition with no treatment, some people still want that information and may live their lives differently as a result. Some consumer advocates argue that it is not up to the medical profession to decide if genetic information is useful or not.

"Is that a decision for someone else to make for me?" says Bloss. "Shouldn't I be able to decide if that information is useful? Those types of arguments are being made." — Roger Collier, CMAJ

CMAJ 2012. DOI:10.1503/cmaj.109-4142

\section{Who should hold the keys to your DNA?}

$\mathrm{C}$ an you handle the truth? Do you really want to know the secrets hidden beneath your skin, deep down in your DNA? There might be bad news in there, lurking in your genes, concealed in your proteins, skulking in your chromosomes.

These are questions more people will likely face as direct-to-consumer genetic tests fall in price and increase in sophistication. Many physicians and geneticists believe consumers shouldn't be answering those questions alone, suggesting that regulations are necessary to ensure medical professionals are part of the process. Critics of regulation disagree, claiming that doctors are being paternalistic and that consumers deserve uninhibited access to their own genetic information.

In the early days of retail genomics, more than a decade ago, there was much concern in the medical community that consumers would be unprepared to receive potentially devastating health news from genetic tests and would suffer great anxiety. That doesn't appear to have transpired, though it was not absurd, at the time, to think that it could, says Timothy Caulfield, a Canada Research Chair in Health Law and Technology.

"It was reasonable speculation. The thinking at the time was that people were going to be getting this information and it was going to be powerful. There would be anxiety. Perhaps people would make inappropriate health care decisions. To some degree, this drove the development of the whole field of genetic counselling," says Caulfield, who teaches in the Faculty of Law and the School of Public Health at the University of Alberta in Edmonton. "It turns out, and data is emerging to support this, that people don't get that anxious about it. Maybe a cohort of people does but, in general, there aren't those high levels of anxiety that people expected."

Prior to the proliferation of direct-toconsumer genetic tests, a systematic review of scientific literature about the mental hazards of genetic discovery uncovered few studies worth reading, though it was suggested that "those undergoing predictive genetic testing do not experience adverse psychological consequences" (Eur J Hum Genet 2000; 8:731-8). Little has changed in the ensuing dozen years, according to a recent study which found that consumers who purchase genetic tests suffer few anxiety symptoms. Over $90 \%$ of participants who completed follow-up reporting indicated that they suffered no test-related distress (N Engl J Med 2011;364:524-34).

"This type of test has been and remains extremely controversial for a variety of reasons. One is the direct-toconsumer nature of it. You get results without a physician and without a genetic counsellor," says Cinnamon Bloss, lead author of the more recent study and lead investigator for the Scripps Genomic Health Initiative at the Scripps Translational Science Institute in La Jolla, California. "Some people feel it is a medical test and that it should be interpreted by a health care professional, because people might see their test results and have anxiety if they don't understand them. Based on our data, people aren't having strong negative reactions in terms of anxiety."

In fact, many people indicate they would take predictive genetic tests "even in the absence of direct treatment consequences" and are "willing to pay reasonably large amounts for the opportunity," according to a random survey of 1463 